\title{
An alternative to the Kelvin decomposition for plane anisotropic elasticity
}

\author{
Boris Desmorat $^{1,2}$, Paolo Vannucci ${ }^{3}$
}

March 3, 2014

${ }^{1}$ Institut Jean Le Rond d'Alembert, CNRS UMR7190 / UPMC Univ Paris 6, Paris France

${ }^{2}$ Université Paris-Sud 11, Orsay - France.

${ }^{3}$ LMV - Laboratoire de Mathématiques de Versailles, UMR 8100 - Université Versailles St Quentin, Versailles - France.

\begin{abstract}
In this paper we propose an alternative tensorial decomposition to the Kelvin's one (introduced by Kelvin in 1856) for plane anisotropic elasticity using the polar formalism (introduced by Verchery in 1979). In a first part of the paper, a parallel between the two approaches is proposed. Thanks to it, some new results are found ; namely, the projectors introduced have a direct interpretation in terms of material symmetry and are intrinsic for any type of symmetry considered, i.e. they do not depend on any elastic modulus for any type of symmetry, unlike in the Kelvin decomposition. The introduction of what we call, in the paper, the polar projectors, stresses and strains gives a new insight into the polar formalism. The results proposed in this paper will hopefully be useful in some cases, for example in the modeling of anisotropic damage evolution in solids.
\end{abstract}

Keywords: Kelvin decomposition; Anisotropy; Polar formalism; Elastic invariants

\section{Introduction}

The classical Hooke's law

$$
\sigma=\mathbb{C} \varepsilon
$$

can be written in a matrix form, exploiting the tensor symmetries of $\sigma, \varepsilon$ and $\mathbb{C}$. We are concerned in this paper with planar elasticity; using a formalism originally due to Kelvin, [1], we can write eq. (1) in the form

$$
\left\{\begin{array}{c}
\sigma_{11} \\
\sigma_{22} \\
\sqrt{2} \sigma_{12}
\end{array}\right\}=\left[\begin{array}{ccc}
C_{1111} & C_{1122} & \sqrt{2} C_{1112} \\
C_{1122} & C_{2222} & \sqrt{2} C_{2212} \\
\sqrt{2} C_{1112} & \sqrt{2} C_{2212} & 2 C_{1212}
\end{array}\right]\left\{\begin{array}{c}
\varepsilon_{11} \\
\varepsilon_{22} \\
\sqrt{2} \varepsilon_{12}
\end{array}\right\} .
$$


In this way, $\sigma$ and $\varepsilon$ are transformed into vectors and $\mathbb{C}$ into a square symmetric matrix of $\mathscr{R}^{3}$. True advantages of the Kelvin formalism on the more used Voigt's one [2], are that $\mathbb{C}$ and its inverse $\mathbb{S}$ are transformed in the same way by a rotation and that the matrix in eq. (2) algebraically corresponds to a symmetric second rank tensor, [3], [4].

Equation (2), by virtue of the spectral theorem for symmetric tensors, [5], can be rewritten in such a manner that the matrix becomes a diagonal one. This possibility was introduced the first by Kelvin as early as 1856, far before the development of modern tensor algebra. Perhaps because too in advance with time, his pioneer work fell almost completely in the oblivion, until some researchers reveal it again to the scientific community in some rather recent works: starting from the eighties, Rychlewsky [6], Mehrabadi and Cowin [3], François [7], R. Desmorat and Marull [8] and more recently de Saxcé and Vallée [4], have used the Kelvin decomposition of elasticity to different purposes.

The Kelvin decomposition of the elastic moduli is particularly important in anisotropic elasticity. In fact, while all the components of $\mathbb{C}$ are frame dependent, i.e. they are not intrinsic quantities describing the mechanical behavior of the material, the eigenvalues of the matrix in eq. (2) are tensor invariants (their value, function of the components of the matrix representing $\mathbb{C}$ in a given frame, do not change under a frame rotation), though, of course, they do not form a complete set of independent invariants (in planar elasticity, there are five independent invariants of $\mathbb{C}$ ).

More recently, another representation of planar elasticity has been proposed by Verchery [9]: the polar formalism. Its very origin is a complex variable transformation, leading to a method for systematically finding all the invariants and syzigy relations of a given tensor, so as to find a complete set of independent invariants. Nevertheless, and unlike in several other approaches that can be found in the literature, all of them are not polynomial invariants. This fact, contrarily to what can be thought, is not a drawback. In fact, the very question in anisotropy is: what are the best elastic moduli to be used? More properly: among the different independent invariants that can be used to represent a tensor, what have a special and interesting meaning for applications? It has been shown that the polar invariants introduced by Verchery are directly linked to the symmetries of the mechanical behavior and to a particularly interesting decomposition of the elastic energy. These facts have been widely and successfully exploited in a recent past, and have brought a new and different insight in the matter of planar anisotropic elasticity, [10], [11], [12], [13], [14].

It seems natural to compare the two methods above, the Kelvin decomposition and the polar formalism, which is the topic of this paper. We proceed hence as follows: the Kelvin decomposition and the main features of the polar formalism are firstly recalled. Then, the Kelvin decomposition is re-interpreted using the polar formalism and finally a new elasticity tensor decomposition is proposed, leading to the definition of what can be called the polar projectors, stresses, strains and energies. A list of the interesting new properties with respect to the Kelvin decomposition is finally presented in the last section, and a potential application to damage mechanics is described. 


\section{The Kelvin decomposition}

As said previously, the idea of the Kelvin decomposition is basically the diagonalization of the matrix representing $\mathbb{C}$ in the Kelvin formalism, eq. (2); the problem is hence the classical one of finding the eigenvalues $\lambda^{i}$ and eigenvectors $\mathbf{E}^{i}$ of matrix $\mathbb{C}$ (here and in the following, an underlined subscript means no summation):

$$
\mathbb{C} \mathbf{E}^{i}=\lambda \stackrel{i}{i} \mathbf{E}^{i}, \quad i=\mathrm{I}, \mathrm{II}, \mathrm{III} .
$$

Eigenvalues $\lambda^{i}$ are homogeneous to a modulus while eigenvectors $\mathbf{E}^{i}$ to a strain. Let us call Kelvin basis the set

$$
\mathscr{E}=\left\{\mathbf{E}^{\mathrm{I}}, \mathbf{E}^{\mathrm{II}}, \mathbf{E}^{\mathrm{III}}\right\} .
$$

By virtue of the spectral theorem, $\mathscr{E}$ is an orthonormal basis; following the suggestion of some authors, e.g. François, [7], [15], the $\lambda^{i}$ will be named Kelvin moduli and the eigenvectors $\mathbf{E}^{i}$, Kelvin modes.

Calling Kelvin projectors the fourth-order dimensionless tensors

$$
\mathbb{P}^{i}=\mathbf{E}^{i} \otimes \mathbf{E}^{i}, \quad \forall i \in\{\mathrm{I}, \mathrm{II}, \mathrm{III}\},
$$

then we get

$$
\mathbb{C}=\lambda^{i} \mathbb{P}^{i}
$$

Each Kelvin projector is represented in the Kelvin formalism by a square singular matrix such that

$$
\mathbb{P}^{\mathrm{I}}+\mathbb{P}^{\mathrm{II}}+\mathbb{P}^{\mathrm{III}}=\mathbb{I}
$$

Calling Kelvin strains and Kelvin stresses respectively each one of the tensors

$$
\varepsilon^{i}=\mathbb{P}^{i} \varepsilon, \sigma^{i}=\mathbb{P}^{i} \sigma \quad \forall i \in\{\mathrm{I}, \mathrm{II}, \mathrm{III}\},
$$

by their same construction, Kelvin strains and stresses are mutually orthogonal:

$$
\varepsilon^{i} \cdot \varepsilon^{j}=0, \quad \sigma^{i} \cdot \sigma^{j}=0 \forall i \neq j \in\{I, I I, I I I\} .
$$

The Hooke's law (1) in the basis $\mathscr{E}$ is hence

$$
\sigma=\lambda^{i} \varepsilon^{i}
$$

and we get

$$
\varepsilon^{i}=\frac{1}{\lambda \underline{\underline{i}}} \sigma^{\underline{i}}, \quad \sigma^{i}=\lambda^{\underline{i}} \varepsilon^{\underline{i}} \forall i \in\{\mathrm{I}, \mathrm{II}, \mathrm{III}\},
$$

Let us consider now the strain energy $W_{s}$ stored in an elastic body:

$$
W_{s}=\frac{1}{2} \varepsilon \cdot \mathbb{C} \varepsilon=\frac{1}{2} \lambda^{i} \varepsilon \cdot \mathbb{P}^{i} \varepsilon=\frac{1}{2} \lambda^{i} \varepsilon^{i} \cdot \varepsilon^{i}
$$

$W_{s}$ can hence be decomposed into three terms $W_{s}^{i}$,

$$
W_{s}^{i}=\frac{1}{2} \lambda \lambda^{\underline{i}} \varepsilon^{\underline{i}} \cdot \varepsilon^{-} \quad \forall i \in\{\mathrm{I}, \mathrm{II}, \mathrm{III}\},
$$


each one of these three terms being associated to the corresponding Kelvin mode. For this reason, we will denote them as Kelvin modal energies. The same procedure can be applied verbatim also to the stress (complementary) energy,

$$
W_{c}=\frac{1}{2} \sigma \cdot \mathbb{S} \sigma
$$

\section{The polar method: basic equations}

In the polar formalism, the Cartesian components of tensor $\mathbb{C}$, in a frame turned counterclockwise by an angle $\theta$ with respect to the $x_{1}$ axis, are expressed as

$$
\begin{aligned}
& C_{1111}(\theta)=T_{0}+2 T_{1}+R_{0} \cos 4\left(\Phi_{0}-\theta\right)+4 R_{1} \cos 2\left(\Phi_{1}-\theta\right), \\
& C_{1112}(\theta)=R_{0} \sin 4\left(\Phi_{0}-\theta\right)+2 R_{1} \sin 2\left(\Phi_{1}-\theta\right), \\
& C_{1122}(\theta)=-T_{0}+2 T_{1}-R_{0} \cos 4\left(\Phi_{0}-\theta\right), \\
& C_{1212}(\theta)=T_{0}-R_{0} \cos 4\left(\Phi_{0}-\theta\right), \\
& C_{1222}(\theta)=-R_{0} \sin 4\left(\Phi_{0}-\theta\right)+2 R_{1} \sin 2\left(\Phi_{1}-\theta\right), \\
& C_{2222}(\theta)=T_{0}+2 T_{1}+R_{0} \cos 4\left(\Phi_{0}-\theta\right)-4 R_{1} \cos 2\left(\Phi_{1}-\theta\right) .
\end{aligned}
$$

The polar moduli $T_{0}, T_{1}, R_{0}$ and $R_{1}$, along with the difference $\Phi_{0}-\Phi_{1}$ of the polar angles are tensor invariants. It appears clearly from eq. (15) that the polar formalism splits each Cartesian component into its isotropic, $T_{0}$ and $T_{1}$, and anisotropic parts, $R_{0}$, $R_{1}$ and $\Phi_{0}-\Phi_{1}$.

By inverting eqs. (15), the expressions of the polar invariants can be obtained:

$$
\begin{aligned}
& T_{0}= \frac{\left[C_{1111}(\theta)-2 C_{1122}(\theta)+4 C_{1212}(\theta)+C_{2222}(\theta)\right]}{8}, \\
& T_{1}= \frac{\left[C_{1111}(\theta)+2 C_{1122}(\theta)+C_{2222}(\theta)\right]}{8}, \\
& R_{0}= \frac{\sqrt{\left[C_{1111}(\theta)-2 C_{1122}(\theta)-4 C_{1212}(\theta)+C_{2222}(\theta)\right]^{2}+16\left[C_{1112}(\theta)-C_{1222}(\theta)\right]^{2}}}{8}, \\
& R_{1}=\frac{\sqrt{\left[C_{1111}(\theta)-C_{2222}(\theta)\right]^{2}+4\left[C_{1112}(\theta)+C_{1222}(\theta)\right]^{2}}}{8}, \\
& \cos 4\left(\Phi_{0}-\Phi_{1}\right)= {\left[C_{1111}(\theta)-2 C_{1122}(\theta)-4 C_{1212}(\theta)+C_{2222}(\theta)\right] } \\
&\left\{\left[C_{1111}(\theta)-C_{2222}(\theta)\right]^{2}-4\left[C_{1112}(\theta)+C_{1222}(\theta)\right]^{2}\right\}+ \\
&+ 16\left[C_{1111}(\theta)-C_{2222}(\theta)\right]\left[C_{1112}^{2}(\theta)-C_{1222}^{2}(\theta)\right] ;
\end{aligned}
$$

the above equations show that both the isotropy moduli $T_{0}$ and $T_{1}$ are linear invariants, the anisotropy moduli $R_{0}$ and $R_{1}$ are the square roots of quadratic invariants while the angular difference $\Phi_{0}-\Phi_{1}$ is a function of a cubic invariant. Hence, only $T_{0}$ and $T_{1}$ are polynomial invariants, among the five polar ones.

For the compliance tensor $\mathbb{S}$, the inverse of $\mathbb{C}$, relations similar to those in eqs. (15) and (16) exist; usually, in this case lower case letters are used for the polar parameters $\left(t_{0}, t_{1}, r_{0}, r_{1}, \varphi_{0}\right.$ and $\left.\varphi_{1}\right)$. 
The polar anisotropic invariants define intrinsically all the possible symmetries of the elastic behavior: it is known that the condition for ordinary orthotropy is linked to a third-order invariant, and in the polar formalism this gets a very simple expression:

$$
\Phi_{0}-\Phi_{1}=K \frac{\pi}{4}, K=\{0,1\}
$$

the value of the invariant $K$ determines the shape of the ordinary orthotropy, and it has a strong role in determining the solution in several optimization problems concerning orthotropic materials. Actually, for the same set of polar moduli $T_{0}, T_{1}, R_{0}$ and $R_{1}$, two distinct orthotropic materials can exist, one with $K=0$, the other one with $K=1$, whose mechanical properties are different.

Besides condition (17), it is simple to check that orthotropy can be obtained in eq. (15) also when

$$
R_{0}=0
$$

or

$$
R_{1}=0 \text {. }
$$

Both these cases are special orthotropies, determined by a condition on a quadratic invariant; in particular, the last condition corresponds to the well known 3D case of cubic syngony, the so-called square symmetry, characterized by a periodicity of $\pi / 2$ of the Cartesian components, while the first one is a the so-called $R_{0}$-orthotropy, a special case of orthotropy discovered by the aid of the polar method, [11], in the plane case, and confirmed by Forte for the 3D case, [16]. We remark that there are five independent and non null invariants for the $K=1$ ordinary orthotropy, four for the $K=0$ case and three for the two special orthotropies.

Finally, isotropy is stated by the two simultaneous intrinsic conditions (18) and (19) and can be interpreted as the simultaneous presence of two special orthotropies.

The above conditions for the orthotropy of $\mathbb{C}$ can be repeated verbatim for $\mathbb{S}$, simply replacing the uppercase with lowercase letters. Nevertheless, while condition (19) implies the same for $r_{1}$, condition (18) does not imply that $r_{0}=0$. This fact, by reciprocity, implies the independent existence of another case of symmetry of the elastic behaviour, the $r_{0}$-orthotropy, concerning $\mathbb{S}$, but not $\mathbb{C}$, [13], just as $R_{0}$-orthotropy concerns $\mathbb{C}$ but not $\mathbb{S}$. In particular, it can be proved that for $r_{0}$-orthotropic materials it is

$$
R_{0}=\frac{R_{1}^{2}}{T_{1}}, K=1 ;
$$

so the number of independent non null invariants is four for $\mathbb{C}$, and three for $\mathbb{S}$.

The polar formalism apply as well to tensors of any rank; we are concerned here also with the polar representation of $\sigma$ and $\varepsilon$ :

$$
\begin{array}{ll}
\sigma_{11}=T+R \cos 2(\Phi-\theta), & \varepsilon_{11}=t+r \cos 2(\varphi-\theta), \\
\sigma_{22}=T-R \cos 2(\Phi-\theta), & \varepsilon_{22}=t-r \cos 2(\varphi-\theta), \\
\sigma_{12}=R \sin 2(\Phi-\theta), & \varepsilon_{12}=r \sin 2(\varphi-\theta) ;
\end{array}
$$


$T$ and $R$ are invariants, while $\Phi$ is an angle fixing the frame; their expression is

$$
\begin{aligned}
& T=\frac{\sigma_{11}(\theta)+\sigma_{22}(\theta)}{2}, \\
& R=\sqrt{\left[\frac{\sigma_{11}(\theta)-\sigma_{22}(\theta)}{2}\right]^{2}+\sigma_{12}^{2}(\theta)}, \\
& \tan 2 \Phi=\frac{2 \sigma_{12}(\theta)}{\sigma_{11}(\theta)-\sigma_{22}(\theta)} .
\end{aligned}
$$

Similar results are valid also for the polar parameters of $\varepsilon: t, r$ and $\varphi$. A last remark: it is apparent from eqs. (21) and (22) that the polar formalism for second rank symmetric tensors is nothing but the algebraic translation of the Mohr's circle.

The strain energy $W_{s}$ takes the following form with the polar formalism, [10]

$$
W_{s}=4 T_{1} t^{2}+8 R_{1} t r \cos 2\left(\Phi_{1}-\varphi\right)+2\left[R_{0} \cos 4\left(\Phi_{0}-\varphi\right)+T_{0}\right] r^{2} .
$$

In this formula, we can see that $T_{1}$ acts only on the spherical part $t$ of $\varepsilon, T_{0}$ and $R_{0}$ only on the deviatoric part $r$, and $R_{1}$ on both. Using this result, it can be shown that condition (19) is the minimal requirement for splitting $W_{s}$ into two separated parts, $W_{s}^{s p h}$, the spherical part, and $W_{s}^{d e v}$, the deviatoric one, depending, the first, only upon the spherical parts of $\sigma$ and $\varepsilon$ ( $T$ and $t$ respectively), the second only upon the deviatoric parts ( $R$ and $r$ respectively).

To end this section, we remark that the above polar invariants (16) are completely equivalent to those found by de Saxcé and Vallée, following an approach starting from the Kelvin decomposition. Actually, the invariants given in eqs. (54) to (59) of [4], can be shown to be equal to

$$
\lambda=2 T_{1}-T_{0}, \quad \mu=T_{0}, I_{2}^{2}=16 R_{1}^{2}, I_{4}^{2}=R_{0}^{2}, \quad-\frac{\zeta_{i}}{\zeta_{r}}=\tan 4\left(\Phi_{0}-\Phi_{1}\right) .
$$

\section{The Kelvin decomposition with the polar formalism}

We use now the polar formalism to express the Kelvin moduli, modes, projectors, strains and strain energy. The procedure is almost straightforward: relations (15) are introduced into the matrix in eq. (2) to express the Cartesian components $C_{i j k l}$ of $\mathbb{C}$ by the polar formalism. Then, the type of symmetry, if any, is introduced using conditions (17), (18), (19) or (20). The eigenvalues $\lambda^{i}$ and eigenvectors $\mathbf{E}^{i}$ of the matrix in eq. (2) are so calculated and they are of course given as functions of the polar parameters of $\mathbb{C}$. The Kelvin projectors $\mathbb{P}^{i}$, strains $\varepsilon^{i}$ and modal energies $W^{i}$ are then computed (the polar stresses, not given here for the sake of shortness, have the same expression of the polar strains, and can be obtained simply swapping upper- and lower-case letters for all of the polar parameters appearing in the expressions of the polar strains). The polar formalism allows hence to distinguish the results upon the type of the elastic symmetry and to express all the quantities as functions of intrinsic quantities, the polar invariants.

We give here the results for the different types of orthotropies and for isotropy; the general case of totally anisotropic material can be formulated, but it is not given here, because of its complexity (the solution of the characteristic equation to find the 
eigenvalues is not simple in such a case). For the sake of simplicity, and without loss of generality, in the following we will put $\Phi_{1}=0$ in eq. (2) to fix the frame. Also, in order to give a simpler form to the results, let us introduce the following two modules, that are invariants too:

$$
A=T_{0}-2 T_{1}+(-1)^{K} R_{0}, \quad B=\sqrt{16 R_{1}^{2}+A^{2}} .
$$

\subsection{Ordinary orthotropy}

If eq. (17) is introduced into eq. (2), then we get:

- Kelvin moduli

$$
\lambda^{\mathrm{I}}=2\left[T_{0}-(-1)^{K} R_{0}\right], \quad \lambda^{\mathrm{II}}=A-B+4 T_{1}, \quad \lambda^{\mathrm{III}}=A+B+4 T_{1} .
$$

- Kelvin modes

$$
\begin{gathered}
\mathbf{E}^{\mathrm{I}}=\left\{\begin{array}{l}
0 \\
0 \\
1
\end{array}\right\}, \\
\mathbf{E}^{\mathrm{II}}=\frac{|A|}{\sqrt{A^{2}+\left(B-4 R_{1}\right)^{2}}}\left\{\begin{array}{c}
\frac{B-4 R_{1}}{A} \\
1 \\
0
\end{array}\right\}, \\
\mathbf{E}^{\mathrm{III}}=\frac{|A|}{\sqrt{A^{2}+\left(B+4 R_{1}\right)^{2}}}\left\{\begin{array}{c}
-\frac{B+4 R_{1}}{A} \\
1 \\
0
\end{array}\right\} .
\end{gathered}
$$

- Kelvin projectors

$$
\begin{gathered}
\mathbb{P}^{\mathrm{I}}=\left[\begin{array}{lll}
0 & 0 & 0 \\
0 & 0 & 0 \\
0 & 0 & 1
\end{array}\right], \\
\mathbb{P}^{\mathrm{II}}=\frac{1}{A^{2}+\left(B-4 R_{1}\right)^{2}}\left[\begin{array}{ccc}
\left(B-4 R_{1}\right)^{2} & A\left(B-4 R_{1}\right) & 0 \\
A\left(B-4 R_{1}\right) & A^{2} & 0 \\
0 & 0 & 0
\end{array}\right], \\
\mathbb{P}^{\mathrm{III}}=\frac{1}{A^{2}+\left(B+4 R_{1}\right)^{2}}\left[\begin{array}{ccc}
\left(B+4 R_{1}\right)^{2} & -A\left(B+4 R_{1}\right) & 0 \\
-A\left(B+4 R_{1}\right) & A^{2} & 0 \\
0 & 0 & 0
\end{array}\right] .
\end{gathered}
$$


- Kelvin strains

$$
\begin{gathered}
\varepsilon^{\mathrm{I}}=\sqrt{2} r \sin 2 \varphi\left\{\begin{array}{l}
0 \\
0 \\
1
\end{array}\right\}, \\
\varepsilon^{\mathrm{II}}=\frac{\left(A+B-4 R_{1}\right) t-\left(A-B+4 R_{1}\right) r \cos 2 \varphi}{A^{2}+\left(B-4 R_{1}\right)^{2}}\left\{\begin{array}{c}
B-4 R_{1} \\
A \\
0
\end{array}\right\}, \\
\varepsilon^{\mathrm{III}}=\frac{\left(-A+B+4 R_{1}\right) t-\left(A+B+4 R_{1}\right) r \cos 2 \varphi}{A^{2}+\left(B+4 R_{1}\right)^{2}}\left\{\begin{array}{c}
B+4 R_{1} \\
-A \\
0
\end{array}\right\} .
\end{gathered}
$$

- Kelvin modal energies

$$
\begin{gathered}
W_{s}^{\mathrm{I}}=2\left[T_{0}-(-1)^{K} R_{0}\right] r^{2} \sin ^{2} 2 \varphi, \\
W_{s}^{\mathrm{II}}=\frac{\left(B+4 R_{1}\right)\left(A-B+4 T_{1}\right)}{4 A^{2} B}\left[\left(A+B-4 R_{1}\right) t-\left(A-B+4 R_{1}\right) r \cos 2 \varphi\right]^{2}, \\
W_{s}^{\mathrm{III}}=\frac{\left(B-4 R_{1}\right)\left(A+B+4 T_{1}\right)}{4 A^{2} B}\left[\left(-A+B+4 R_{1}\right) t+\left(A+B+4 R_{1}\right) r \cos 2 \varphi\right]^{2} .
\end{gathered}
$$

- Total strain energy

$$
W_{s}=W_{s}^{\mathrm{I}}+W_{s}^{\mathrm{II}}+W_{s}^{\mathrm{III}}=2 T_{0} r^{2}+4 T_{1} t^{2}+2(-1)^{K} R_{0} r^{2} \cos 4 \varphi+8 R_{1} t r \cos 2 \varphi,
$$

which of course is the corresponding of (23) for the case of ordinarily orthotropic materials.

\section{$4.2 R_{0}$-orthotropy}

Inserting condition (18) into eq. (2) leaves unchanged almost all the results found for ordinary orthotropy; the only changes are:

$$
R_{0}=0 \rightarrow\left\{\begin{aligned}
A & =T_{0}-2 T_{1}, \\
\lambda^{\mathrm{I}} & =2 T_{0}, \\
W_{s}^{\mathrm{I}} & =2 T_{0} r^{2} \sin ^{2} 2 \varphi, \\
W_{s} & =2 T_{0} r^{2}+4 T_{1} t^{2}+8 R_{1} t r \cos 2 \varphi .
\end{aligned}\right.
$$




\section{$4.3 \quad r_{0}$-orthotropy}

If conditions (20) are introduced into eq. (2), then the only changes in the results found for ordinary orthotropy are

$$
r_{0}=0 \rightarrow\left\{\begin{array}{l}
R_{0}=\frac{R_{1}^{2}}{T_{1}}, K=1, \\
A=T_{0}-2 T_{1}-\frac{R_{1}^{2}}{T_{1}}, \\
W_{s}^{\mathrm{I}}=2\left(T_{0}-\frac{R_{1}^{2}}{T_{1}}\right) r^{2} \sin ^{2} 2 \varphi, \\
W_{s}=2 T_{0} r^{2}+4 T_{1} t^{2}+2 \frac{R_{1}^{2}}{T_{1}} r^{2} \cos 4 \varphi+8 R_{1} \operatorname{tr} \cos 2 \varphi .
\end{array}\right.
$$

\subsection{Square symmetry $\left(R_{1}=0\right)$}

This is a particularly interesting case, because, unlike all the previous cases, the eigenvectors $\mathbf{E}^{i}$, and by consequence the Kelvin projectors $\mathbb{P}^{i}$, do not depend upon the elastic moduli: all the square symmetric plies share the same $\mathbf{E}^{i}$ and $\mathbb{P}^{i}$. We list below all the results for this case:

- Kelvin moduli

$$
\lambda^{\mathrm{I}}=2\left(T_{0}-R_{0}\right), \quad \lambda^{\mathrm{II}}=2\left(T_{0}+R_{0}\right), \quad \lambda^{\mathrm{III}}=4 T_{1} .
$$

- Kelvin modes

$$
\mathbf{E}^{\mathrm{I}}=\left\{\begin{array}{l}
0 \\
0 \\
1
\end{array}\right\}, \quad \mathbf{E}^{\mathrm{II}}=\frac{1}{\sqrt{2}}\left\{\begin{array}{r}
-1 \\
1 \\
0
\end{array}\right\}, \quad \mathbf{E}^{\mathrm{III}}=\frac{1}{\sqrt{2}}\left\{\begin{array}{l}
1 \\
1 \\
0
\end{array}\right\} .
$$

- Kelvin projectors

$$
\mathbb{P}^{\mathrm{I}}=\left[\begin{array}{lll}
0 & 0 & 0 \\
0 & 0 & 0 \\
0 & 0 & 1
\end{array}\right], \mathbb{P}^{\mathrm{II}}=\frac{1}{2}\left[\begin{array}{rrr}
1 & -1 & 0 \\
-1 & 1 & 0 \\
0 & 0 & 0
\end{array}\right], \quad \mathbb{P}^{\mathrm{III}}=\frac{1}{2}\left[\begin{array}{lll}
1 & 1 & 0 \\
1 & 1 & 0 \\
0 & 0 & 0
\end{array}\right]
$$

- Kelvin strains

$$
\varepsilon^{\mathrm{I}}=\sqrt{2} r \sin 2 \varphi\left\{\begin{array}{l}
0 \\
0 \\
1
\end{array}\right\}, \quad \varepsilon^{\mathrm{II}}=r \cos 2 \varphi\left\{\begin{array}{r}
1 \\
-1 \\
0
\end{array}\right\}, \quad \varepsilon^{\mathrm{III}}=t\left\{\begin{array}{l}
1 \\
1 \\
0
\end{array}\right\} .
$$

- Kelvin modal energies

$$
W_{s}^{\mathrm{I}}=2\left(T_{0}-R_{0}\right) r^{2} \sin ^{2} 2 \varphi, W_{s}^{\mathrm{II}}=2\left(T_{0}+R_{0}\right) r^{2} \cos ^{2} 2 \varphi, W_{s}^{\mathrm{III}}=4 T_{1} t^{2} .
$$

- Total strain energy

$$
W_{s}=2 T_{0} r^{2}+4 T_{1} t^{2}+2 R_{0} r^{2} \cos 4 \varphi
$$




\subsection{Isotropy}

Now, conditions (18) and (19) must be inserted into eq. (2); the Kelvin modes, projectors and strains are the same of the previous case, while:

- Kelvin moduli

$$
\lambda^{\mathrm{I}}=\lambda^{\mathrm{II}}=2 T_{0}, \quad \lambda^{\mathrm{III}}=4 T_{1} .
$$

Because of the existence of a double eigenvalue $\lambda^{\mathrm{I}}=\lambda^{\mathrm{II}}$, it is convenient to introduce only two Kelvin projectors, two Kelvin strains and two modal energies.

- Kelvin projectors

$$
\mathbb{P}^{\mathrm{I} / \mathrm{II}}=\mathbb{P}^{\mathrm{I}}+\mathbb{P}^{\mathrm{II}}=\frac{1}{2}\left[\begin{array}{rrr}
1 & -1 & 0 \\
-1 & 1 & 0 \\
0 & 0 & 2
\end{array}\right], \quad \mathbb{P}^{\mathrm{III}}=\frac{1}{2}\left[\begin{array}{lll}
1 & 1 & 0 \\
1 & 1 & 0 \\
0 & 0 & 0
\end{array}\right]
$$

- Kelvin strains

$$
\varepsilon^{\mathrm{I} / \mathrm{II}}=r\left\{\begin{array}{r}
\cos 2 \varphi \\
-\cos 2 \varphi \\
\sqrt{2} \sin 2 \varphi
\end{array}\right\}, \quad \varepsilon^{\mathrm{III}}=t\left\{\begin{array}{l}
1 \\
1 \\
0
\end{array}\right\}
$$

$\varepsilon^{\mathrm{I} / \mathrm{II}}$ and $\varepsilon^{\mathrm{III}}$ are nothing else than the deviatoric and spherical parts of the strain tensor.

- Kelvin modal energies

$$
W_{s}^{\mathrm{I} / \mathrm{II}}=2 T_{0} r^{2}, \quad W_{s}^{\mathrm{III}}=4 T_{1} t^{2} .
$$

- Total strain energy

$$
W_{s}=2 T_{0} r^{2}+4 T_{1} t^{2}
$$

\section{An alternative formalism: the polar decomposition}

We introduce now a new formalism for plane elasticity, inspired by the Kelvin decomposition but based upon the polar formalism. The way we find it is rather easy: eq. (15) shows that the matrix representing tensor $\mathbb{C}$ in the Kelvin formalism, eq. (2), can be written as

$$
\mathbb{C}(\theta)=T_{0} \mathbb{T}_{0}+2 T_{1} \mathbb{T}_{1}+R_{0 c} \mathbb{R}_{0 c}+R_{0 s} \mathbb{R}_{0 s}+2 R_{1} \mathbb{R}_{1},
$$

where, for the sake of simplicity and without loss of generality, we have fixed a frame choosing $\Phi_{1}=0$, and put

$$
R_{0} \cos 4 \Phi_{0}=R_{0 c}, \quad R_{0} \sin 4 \Phi_{0}=R_{0 s} .
$$


It is immediately recognized that $R_{0 c}$ and $R_{0 s}$ are tensor invariants too. If the material is ordinarily orthotropic, then, see eq. (17) and recall that we have chosen $\Phi_{1}=0$,

$$
\Phi_{0}-\Phi_{1}\left(=\Phi_{0}\right)=K \frac{\pi}{4}, K=0,1 \rightarrow R_{0 c}=(-1)^{K} R_{0}, R_{0 s}=0,
$$

while the invariant $R_{0 c}$ vanishes whenever

$$
\Phi_{0}-\Phi_{1}\left(=\Phi_{0}\right)=\frac{\pi}{8}+\kappa \frac{\pi}{4}, \kappa \in \mathbb{N} \rightarrow R_{0 c}=0 .
$$

Finally, the particular case of $r_{0}$-orthotropy gives, see eqs. (20) and (47),

$$
R_{0 c}=-\frac{R_{1}^{2}}{T_{1}}, R_{0 s}=0 .
$$

In eq. (45), $\mathbb{T}_{0}, \mathbb{T}_{1}, \mathbb{R}_{0 c}, \mathbb{R}_{0 s}$ and $\mathbb{R}_{1}$ are fourth-rank tensors of $\mathscr{R}^{2}$ of the type of elasticity, defined by (the set $\left\{\mathbf{e}_{1}, \mathbf{e}_{2}\right\}$ is an orthonormal basis of $\mathscr{R}^{2}$ )

$$
\begin{gathered}
\mathbb{T}_{0}=\left(\mathbf{e}_{1} \otimes \mathbf{e}_{1}-\mathbf{e}_{2} \otimes \mathbf{e}_{2}\right) \otimes\left(\mathbf{e}_{1} \otimes \mathbf{e}_{1}-\mathbf{e}_{2} \otimes \mathbf{e}_{2}\right)+ \\
\left(\mathbf{e}_{1} \otimes \mathbf{e}_{2}+\mathbf{e}_{2} \otimes \mathbf{e}_{1}\right) \otimes\left(\mathbf{e}_{1} \otimes \mathbf{e}_{2}+\mathbf{e}_{2} \otimes \mathbf{e}_{1}\right), \\
\mathbb{T}_{1}=\left(\mathbf{e}_{1} \otimes \mathbf{e}_{1}+\mathbf{e}_{2} \otimes \mathbf{e}_{2}\right) \otimes\left(\mathbf{e}_{1} \otimes \mathbf{e}_{1}+\mathbf{e}_{2} \otimes \mathbf{e}_{2}\right), \\
\mathbb{R}_{0 c}=c_{4}\left[\left(\mathbf{e}_{1} \otimes \mathbf{e}_{1}-\mathbf{e}_{2} \otimes \mathbf{e}_{2}\right) \otimes\left(\mathbf{e}_{1} \otimes \mathbf{e}_{1}-\mathbf{e}_{2} \otimes \mathbf{e}_{2}\right)-\right. \\
\left.\left(\mathbf{e}_{1} \otimes \mathbf{e}_{2}+\mathbf{e}_{2} \otimes \mathbf{e}_{1}\right) \otimes\left(\mathbf{e}_{1} \otimes \mathbf{e}_{2}+\mathbf{e}_{2} \otimes \mathbf{e}_{1}\right)\right]- \\
s_{4}\left[\left(\mathbf{e}_{1} \otimes \mathbf{e}_{1}-\mathbf{e}_{2} \otimes \mathbf{e}_{2}\right) \otimes\left(\mathbf{e}_{1} \otimes \mathbf{e}_{2}+\mathbf{e}_{2} \otimes \mathbf{e}_{1}\right)+\right. \\
\left.\left(\mathbf{e}_{1} \otimes \mathbf{e}_{2}+\mathbf{e}_{2} \otimes \mathbf{e}_{1}\right) \otimes\left(\mathbf{e}_{1} \otimes \mathbf{e}_{1}-\mathbf{e}_{2} \otimes \mathbf{e}_{2}\right)\right], \\
\mathbb{R}_{0 s}=s_{4}\left[\left(\mathbf{e}_{1} \otimes \mathbf{e}_{1}-\mathbf{e}_{2} \otimes \mathbf{e}_{2}\right) \otimes\left(\mathbf{e}_{1} \otimes \mathbf{e}_{1}-\mathbf{e}_{2} \otimes \mathbf{e}_{2}\right)-\right. \\
\left.\left(\mathbf{e}_{1} \otimes \mathbf{e}_{2}+\mathbf{e}_{2} \otimes \mathbf{e}_{1}\right) \otimes\left(\mathbf{e}_{1} \otimes \mathbf{e}_{2}+\mathbf{e}_{2} \otimes \mathbf{e}_{1}\right)\right]+ \\
c_{4}\left[\left(\mathbf{e}_{1} \otimes \mathbf{e}_{1}-\mathbf{e}_{2} \otimes \mathbf{e}_{2}\right) \otimes\left(\mathbf{e}_{1} \otimes \mathbf{e}_{2}+\mathbf{e}_{2} \otimes \mathbf{e}_{1}\right)+\right. \\
\left.\left(\mathbf{e}_{1} \otimes \mathbf{e}_{2}+\mathbf{e}_{2} \otimes \mathbf{e}_{1}\right) \otimes\left(\mathbf{e}_{1} \otimes \mathbf{e}_{1}-\mathbf{e}_{2} \otimes \mathbf{e}_{2}\right)\right], \\
\mathbb{R}_{1}=2 c_{2}\left[\left(\mathbf{e}_{1} \otimes \mathbf{e}_{1}\right) \otimes\left(\mathbf{e}_{1} \otimes \mathbf{e}_{1}\right)-\left(\mathbf{e}_{2} \otimes \mathbf{e}_{2}\right) \otimes\left(\mathbf{e}_{2} \otimes \mathbf{e}_{2}\right)\right]+ \\
s_{2}\left[\left(\mathbf{e}_{1} \otimes \mathbf{e}_{1}+\mathbf{e}_{2} \otimes \mathbf{e}_{2}\right) \otimes\left(\mathbf{e}_{1} \otimes \mathbf{e}_{2}+\mathbf{e}_{2} \otimes \mathbf{e}_{1}\right)+\right. \\
\left.\left(\mathbf{e}_{1} \otimes \mathbf{e}_{2}+\mathbf{e}_{2} \otimes \mathbf{e}_{1}\right) \otimes\left(\mathbf{e}_{1} \otimes \mathbf{e}_{1}+\mathbf{e}_{2} \otimes \mathbf{e}_{2}\right)\right],
\end{gathered}
$$

where, for the sake of conciseness,

$$
\begin{array}{ll}
c_{2}=\cos 2 \theta, & s_{2}=\sin 2 \theta, \\
c_{4}=\cos 4 \theta, & s_{4}=\sin 4 \theta .
\end{array}
$$

Tensors $\mathbb{T}_{0}, \mathbb{T}_{1}, \mathbb{R}_{0 c}, \mathbb{R}_{0 s}$ and $\mathbb{R}_{1}$ play a role similar to that of the Kelvin projectors, eq. (6), in the sense that they give a decomposition of the elasticity tensor $\mathbb{C}$. For this reason we will call them polar projectors and denote them in a general way by $\mathbb{P}_{p o l}^{i}$, i.e.

$$
\mathbb{P}_{p o l}^{i} \in\left\{\mathbb{T}_{1}, \mathbb{T}_{0}, \mathbb{R}_{0 c}, \mathbb{R}_{0 s}, \mathbb{R}_{1}\right\}
$$


Their matrix form is quite simple:

$$
\begin{gathered}
\mathbb{T}_{0}=\left[\begin{array}{rrr}
1 & -1 & 0 \\
-1 & 1 & 0 \\
0 & 0 & 2
\end{array}\right], \mathbb{T}_{1}=\left[\begin{array}{lll}
1 & 1 & 0 \\
1 & 1 & 0 \\
0 & 0 & 0
\end{array}\right], \mathbb{R}_{0 c}=\left[\begin{array}{rrr}
c_{4} & -c_{4} & -\sqrt{2} s_{4} \\
-c_{4} & c_{4} & \sqrt{2} s_{4} \\
-\sqrt{2} s_{4} & \sqrt{2} s_{4} & -2 c_{4}
\end{array}\right], \\
\mathbb{R}_{0 s}=\left[\begin{array}{rrrr}
s_{4} & -s_{4} & \sqrt{2} c_{4} \\
-s_{4} & s_{4} & -\sqrt{2} c_{4} \\
\sqrt{2} c_{4} & -\sqrt{2} c_{4} & -2 s_{4}
\end{array}\right], \mathbb{R}_{1}=\left[\begin{array}{rrr}
2 c_{2} & 0 & \sqrt{2} s_{2} \\
0 & -2 c_{2} & \sqrt{2} s_{2} \\
\sqrt{2} s_{2} & \sqrt{2} s_{2} & 0
\end{array}\right] .
\end{gathered}
$$

A relation similar to eq. (45) holds also for tensor $\mathbb{S}$, it is sufficient to replace the polar stiffness moduli by the corresponding compliance ones, in practice, they must be written with lowercase letters, while the projectors are exactly the same.

The polar projectors have some algebraic properties, listed below:

$$
\begin{aligned}
& \left\|\mathbb{T}_{1}\right\|=2,\left\|\mathbb{T}_{0}\right\|=\left\|\mathbb{R}_{0 c}\right\|=\left\|\mathbb{R}_{0 s}\right\|=\left\|\mathbb{R}_{1}\right\|=2 \sqrt{2}, \\
& \operatorname{det}\left(\mathbb{P}_{p o l}^{i}\right)=0, \\
& \mathbb{P}_{\text {pol }}^{i} \cdot \mathbb{P}_{\text {pol }}^{j}=0, \quad \forall \mathbb{P}_{\text {pol }}^{i} \neq \mathbb{P}_{\text {pol }}^{j} \in\left\{\mathbb{T}_{1}, \mathbb{T}_{0}, \mathbb{R}_{0 c}, \mathbb{R}_{0 s}, \mathbb{R}_{1}\right\} . \\
& \sum_{i} \mathbb{P}_{\text {pol }}^{i} \neq \mathbb{I},
\end{aligned}
$$

So, the polar projectors are singular orthogonal tensors, whose norm is not the unity and whose sum is not the identity. Actually, they constitute another way to decompose an elasticity tensor, that has some particular features and links with the material symmetries, through the polar invariants. It is worth recalling that the coefficients of the linear combination giving the elastic tensor, eq. (45), are tensor invariants and that all the dependence upon the orientation $\theta$ is in the three projectors $\mathbb{R}_{0 c}, \mathbb{R}_{0 s}$ and $\mathbb{R}_{1}$, linked to the anisotropic part, while the two projectors $\mathbb{T}_{0}$ and $\mathbb{T}_{1}$, linked to the isotropic part, are insensible to the orientation. Fixing the orientation to zero gives

$\theta=0 \rightarrow \mathbb{R}_{0 c}=\left[\begin{array}{rrr}1 & -1 & 0 \\ -1 & 1 & 0 \\ 0 & 0 & -2\end{array}\right], \mathbb{R}_{0 s}=\left[\begin{array}{rrr}0 & 0 & \sqrt{2} \\ 0 & 0 & -\sqrt{2} \\ \sqrt{2} & -\sqrt{2} & 0\end{array}\right], \mathbb{R}_{1}=\left[\begin{array}{rrr}2 & 0 & 0 \\ 0 & -2 & 0 \\ 0 & 0 & 0\end{array}\right]$

Some material symmetries, i.e., algebraically speaking, some particular values of the polar invariants, make one or more anisotropic polar invariants vanish, so eliminating the corresponding polar projectors from the sum of tensors giving $\mathbb{C}$. Namely, as already recalled in eq. (47), ordinary orthotropy eliminates $\mathbb{R}_{0 s}$, while condition (48) eliminates $\mathbb{R}_{0 c}$. Also, $R_{0}$-orthotropy, eq. (18), eliminates both $\mathbb{R}_{0 c}$ and $\mathbb{R}_{0 s}$, while square symmetry, eq. (19), $\mathbb{R}_{1}$ and finally, isotropy eliminates all of them.

Just like for the Kelvin decomposition, let us now introduce the tensors

$$
\varepsilon_{p o l}^{i}=\mathbb{P}_{p o l}^{i} \varepsilon, \sigma_{p o l}^{i}=\mathbb{P}_{p o l}^{i} \sigma \quad \forall i \in\{\mathrm{I}, \ldots, \mathrm{V}\},
$$

that we will call polar strains and polar stresses respectively. Using eq. (21), one gets, 
for the case of the polar strains,

$$
\begin{aligned}
& \varepsilon_{p o l}^{\mathrm{I}}=\mathbb{T}_{0} \varepsilon=\left\{\begin{array}{c}
2 r \cos 2(\varphi-\theta) \\
-2 r \cos 2(\varphi-\theta) \\
2 \sqrt{2} r \sin 2(\varphi-\theta)
\end{array}\right\}, \\
& \varepsilon_{p o l}^{\mathrm{II}}=\mathbb{T}_{1} \varepsilon=\left\{\begin{array}{c}
2 t \\
2 t \\
0
\end{array}\right\}, \\
& \varepsilon_{p o l}^{\mathrm{III}}=\mathbb{R}_{0 c} \varepsilon=\left\{\begin{array}{c}
2 r \cos 2(\varphi+\theta) \\
-2 r \cos 2(\varphi+\theta) \\
-2 \sqrt{2} r \sin 2(\varphi+\theta)
\end{array}\right\}, \\
& \varepsilon_{p o l}^{\mathrm{IV}}=\mathbb{R}_{0 s} \varepsilon=\left\{\begin{array}{c}
2 r \sin 2(\varphi+\theta) \\
-2 r \sin 2(\varphi+\theta) \\
2 \sqrt{2} r \cos 2(\varphi+\theta)
\end{array}\right\}, \\
& \varepsilon_{p o l}^{\mathrm{V}}=\mathbb{R}_{1} \varepsilon=\left\{\begin{array}{c}
2 t \cos 2 \theta+2 r \cos 2(\varphi-2 \theta) \\
-2 t \cos 2 \theta+2 r \cos 2(\varphi-2 \theta) \\
2 \sqrt{2} t \sin 2 \theta
\end{array}\right\},
\end{aligned}
$$

If one fixes the direction $\theta=0$, then the above expressions simplify to

$$
\theta=0 \rightarrow\left\{\begin{array}{c}
\varepsilon_{p o l}^{\mathrm{I}}=\mathbb{T}_{0} \varepsilon=\left\{\begin{array}{c}
\varepsilon_{1}-\varepsilon_{2} \\
-\varepsilon_{1}+\varepsilon_{2} \\
2 \varepsilon_{6}
\end{array}\right\}=\left\{\begin{array}{c}
2 r \cos 2 \varphi \\
-2 r \cos 2 \varphi \\
2 \sqrt{2} r \sin 2 \varphi
\end{array}\right\}, \\
\varepsilon_{p o l}^{\mathrm{II}}=\mathbb{T}_{1} \varepsilon=\left\{\begin{array}{c}
\varepsilon_{1}+\varepsilon_{2} \\
\varepsilon_{1}+\varepsilon_{2} \\
0
\end{array}\right\}=\left\{\begin{array}{c}
2 t \\
2 t \\
0
\end{array}\right\}, \\
\varepsilon_{p o l}^{\mathrm{III}}=\mathbb{R}_{0 c} \varepsilon=\left\{\begin{array}{c}
\varepsilon_{1}-\varepsilon_{2} \\
-\varepsilon_{1}+\varepsilon_{2} \\
-2 \varepsilon_{6}
\end{array}\right\}=\left\{\begin{array}{c}
2 r \cos 2 \varphi \\
-2 r \cos 2 \varphi \\
-2 \sqrt{2} r \sin 2 \varphi
\end{array}\right\}, \\
\varepsilon_{p o l}^{\mathrm{IV}}=\mathbb{R}_{0 s} \varepsilon=\left\{\begin{array}{c}
\sqrt{2} \varepsilon_{6} \\
-\sqrt{2} \varepsilon_{6} \\
\sqrt{2}\left(\varepsilon_{1}-\varepsilon_{2}\right)
\end{array}\right\}=\left\{\begin{array}{c}
2 r \sin 2 \varphi \\
-2 r \sin 2 \varphi \\
2 \sqrt{2} r \cos 2 \varphi
\end{array}\right\}, \\
\varepsilon_{p o l}^{\mathrm{V}}=\mathbb{R}_{1} \varepsilon=\left\{\begin{array}{c}
2 \varepsilon_{1} \\
-2 \varepsilon_{2} \\
0
\end{array}\right\}=\left\{\begin{array}{c}
2 t+2 r \cos 2 \varphi \\
-2 t+2 r \cos 2 \varphi \\
0
\end{array}\right\} .
\end{array}\right.
$$

Similar expressions hold also for the polar stresses $\sigma_{p o l}^{i}$, it is sufficient to replace $t, r$ and $\varphi$ by $T, R$ and $\Phi$, respectively. It is worth noting that, unlike Kelvin strains and stresses, the polar strains and stresses are not necessarily orthogonal and they do not decompose strain and stress:

$$
\sum_{i=\mathrm{I}}^{\mathrm{V}} \varepsilon^{i} \neq \varepsilon, \sum_{i=\mathrm{I}}^{\mathrm{V}} \sigma^{i} \neq \sigma .
$$


On the other hand, noticing that

$$
\varepsilon_{p o l}^{\mathrm{I}}=2 \varepsilon^{d e v}, \quad \varepsilon_{p o l}^{\mathrm{II}}=2 \varepsilon^{s p h},
$$

it is possible to decompose strains or stresses with the first two polar strains or stresses:

$$
\begin{aligned}
& \varepsilon=\varepsilon^{s p h}+\varepsilon^{d e v}=\frac{1}{2}\left(\varepsilon_{p o l}^{\mathrm{I}}+\varepsilon_{p o l}^{\mathrm{II}}\right), \\
& \sigma=\sigma^{s p h}+\sigma^{d e v}=\frac{1}{2}\left(\sigma_{p o l}^{\mathrm{I}}+\sigma_{p o l}^{\mathrm{II}}\right) .
\end{aligned}
$$

Unlike the case of $\sigma$ and $\varepsilon$, the stored elastic energy can be perfectly decomposed by the polar stresses or strains. Let us consider, for instance, the strain energy (similar results exist of course also for the stress energy, it is sufficient to change $\varepsilon$ with $\sigma$ and switch all the polar components denoted by lowercase letters to capital letters, and those denoted by capital letters to lowercase letters):

$$
W_{s}=\frac{1}{2} \varepsilon \cdot \mathbb{C} \varepsilon=\frac{1}{2} \varepsilon \cdot\left[T_{0} \mathbb{T}_{0}+2 T_{1} \mathbb{T}_{1}+R_{0 c} \mathbb{R}_{0 c}+R_{0 s} \mathbb{R}_{0 s}+2(-1)^{h} R_{1} \mathbb{R}_{1}\right] \varepsilon .
$$

Using eqs. (56) we can get:

$$
W_{s}=\sum_{i=\mathrm{I}}^{\mathrm{V}} W_{s p o l}^{i},
$$

with

$$
\begin{aligned}
& W_{s p o l}^{i}=\frac{1}{2} \varepsilon \cdot \beta_{p o l}^{i} \varepsilon_{p o l}^{i} \forall \in\{I, \ldots, V\} \rightarrow \\
& W_{s p o l}^{\mathrm{I}}=\frac{1}{2} \varepsilon \cdot T_{0} \varepsilon_{p o l}^{\mathrm{I}}=\frac{1}{2} T_{0}\left[\left(\varepsilon_{1}-\varepsilon_{2}\right)^{2}+2 \varepsilon_{6}^{2}\right]=2 T_{0} r^{2}, \\
& W_{s p o l}^{\mathrm{II}}=\frac{1}{2} \varepsilon \cdot 2 T_{1} \varepsilon_{p o l}^{\mathrm{II}}=T_{1}\left(\varepsilon_{1}+\varepsilon_{2}\right)^{2}=4 T_{1} t^{2}, \\
& W_{s p o l}^{\mathrm{III}}=\frac{1}{2} \varepsilon \cdot R_{0 c} \varepsilon_{p o l}^{\mathrm{III}}=\frac{1}{2} R_{0 c}\left[\left(\varepsilon_{1}-\varepsilon_{2}\right)^{2}-2 \varepsilon_{6}^{2}\right]=2 R_{0 c} r^{2} \cos 4 \varphi, \\
& W_{s p o l}^{\mathrm{IV}}=\frac{1}{2} \varepsilon \cdot R_{0 s} \varepsilon_{p o l}^{\mathrm{IV}}=\sqrt{2} R_{0 s}\left(\varepsilon_{1}-\varepsilon_{2}\right) \varepsilon_{6}=2 R_{0 s} r^{2} \sin 4 \varphi, \\
& W_{s p o l}^{\mathrm{V}}=\frac{1}{2} \varepsilon \cdot 2 R_{1} \varepsilon_{p o l}^{\mathrm{V}}=2 R_{1}\left(\varepsilon_{1}^{2}-\varepsilon_{2}^{2}\right)=8 R_{1} t r \cos 2 \varphi,
\end{aligned}
$$

where we have indicated by $\beta_{p o l}^{i}$ any one of the polar invariants; the values of the $\beta_{p o l}^{i}$ are apparent from eq. (64). The different terms $W_{s \text { pol }}^{i}$ are the polar strain energies; each one of them is linked to a polar modulus and to a part of the strain tensor: $W_{s}^{\mathrm{I}}$ pol , $W_{s p o l}^{\mathrm{III}}$ and $W_{s p o l}^{\mathrm{IV}}$ depends upon the deviatoric part of $\varepsilon, W_{s}^{\mathrm{II}}$ ol upon its spherical part and $W_{s \text { pol }}^{\mathrm{V}}$ on both of them. The sum of the terms in (64) is of course equal to the expression of $W_{s}$ given in (23), how it can be easily checked.

The above polar energies can be further decomposed. Recalling (61), we get

$$
W_{s p o l}^{i}=\frac{1}{2}\left(\varepsilon^{s p h}+\varepsilon^{d e v}\right) \cdot \beta^{i} \varepsilon_{p o l}^{i}=W_{s p o l}^{s p h i}+W_{s p o l}^{d e v i} \forall \in\{I, \ldots, V\},
$$

where

$$
W_{s p o l}^{s p h i}=\frac{1}{2} \varepsilon^{s p h} \cdot \beta^{-} \varepsilon_{p o l}^{\underline{i}}=\frac{1}{4} \varepsilon^{\mathrm{II}} \cdot \beta^{\underline{i}} \varepsilon_{\text {pol }}^{\underline{i}}, \quad W_{s p o l}^{d e v}=\frac{1}{2} \varepsilon^{d e v} \cdot \beta^{\underline{i}} \varepsilon_{p o l}^{\underline{i}}=\frac{1}{4} \varepsilon^{\mathrm{I}} \cdot \beta^{\underline{i}} \varepsilon_{p o l}^{\underline{i}} .
$$


The total number of non null $W_{s p o l}^{s p h i}$ and $W_{s \text { pol }}^{\text {dev } i}$ is not of ten, as one could expect, but only of six; this happens because, as can be easily checked using eq. (57), it is

$$
\varepsilon_{p o l}^{\mathrm{II}} \cdot \varepsilon_{p o l}^{i}=0 \forall i \in\{\mathrm{I}, \mathrm{III}, \mathrm{IV}\}, \quad \varepsilon_{p o l}^{\mathrm{III}} \cdot \varepsilon_{p o l}^{\mathrm{IV}}=0 .
$$

We can hence find

$$
\begin{aligned}
& W_{s p o l}^{s p h \mathrm{I}}=0, \quad W_{s p o l}^{d e v \mathrm{I}}=W_{s p o l}^{\mathrm{I}}=2 T_{0} r^{2}, \\
& W_{s p o l}^{s p h \mathrm{II}}=W_{s p o l}^{\mathrm{II}}=4 T_{1} t^{2}, \quad W_{s p o l}^{d e v \mathrm{II}}=0, \\
& W_{s p o l}^{s p h \mathrm{III}}=0, \quad W_{s p o l}^{d e v \mathrm{III}}=W_{s p o l}^{\mathrm{III}}=2 R_{0 c} r^{2} \cos 4 \varphi, \\
& W_{s p o l}^{s p h \mathrm{IV}}=0, \quad W_{s p o l}^{d e v \mathrm{IV}}=W_{s p o l}^{\mathrm{IV}}=2 R_{0 s} r^{2} \sin 4 \varphi, \\
& W_{s p o l}^{s p h \mathrm{~V}}=W_{s p o l}^{d e v \mathrm{~V}}=\frac{1}{2} W_{s p o l}^{\mathrm{V}}=4 R_{1} t r \cos 2 \varphi .
\end{aligned}
$$

The only polar energy to have both the spherical and deviatoric part non null, and identical, is $W_{s \text { pol }}^{\mathrm{V}}$. This term is more properly a mixt energy because always depending on both the spherical and deviatoric parts of $\varepsilon$. Also,

$$
W_{s p o l}^{\mathrm{I}}>W_{s p o l}^{\mathrm{III}}, \quad W_{s p o l}^{\mathrm{I}}>W_{s p o l}^{\mathrm{IV}} \forall\{r, \varphi\},
$$

because it is always $T_{0}>R_{0}$, [10], and hence, a fortiori, $T_{0}>R_{0 c}$ and $T_{0}>R_{0 s}$, eq. (46).

\section{Conclusion}

The main properties, with respect to the Kelvin decomposition, of the new tensorial decomposition based on the polar formalism introduced in this paper are :

- Similarly to the Kelvin decomposition, the polar decomposition introduces identical polar projectors when considering the stiffness tensor $\mathbb{C}$ or the compliance tensor $\mathbb{C}^{-1}$.

- The polar decomposition has a direct interpretation in terms of material symmetry, which is not the case for the Kelvin decomposition outside the isotropic and square symmetry cases.

- The projectors are intrinsinc in the polar decomposition for any type of symmetry considered. More precisely, the polar projectors do not depend on any modulus for any type of symmetry. This is true in the case of the Kelvin decomposition only for isotropy and square symmetry.

- The dependence of the polar projectors with any global frame considered is explicitly given by the angle $\theta$.

- Two polar projectors are found to be constant with respect to any material behaviour, i.e. to any polar moduli and to the global frame considered. 
- In the isotropic case, the Kelvin and polar decomposition are identical and the projectors are found to be constant.

- In the square symmetry case, only one Kelvin and polar projector is identical. For the Kelvin decomposition, the associated Kelvin stresses and strains are the spherical part, the diagonal of the deviatoric part and the out of diagonal of the devitoric part. For the polar decomposition, the associated polar stresses and strains are the spherical part, the deviatoric part and the deviatoric part with an opposite sign for the out of diagonal terms.

- For any considered symmetry, two of the polar strains (or stresses) are equal to twice the spherical and deviatoric parts, which results in the fact that the strains (or stress) is equal to the sum of the first two polar strains (or stresses).

- If we introduce another decomposition of $\sigma$ that can be obtained using eq. (45) and the Hooke's law (1):

$$
\sigma=\mu_{p o l}^{\mathrm{I}}+2 \mu_{p o l}^{\mathrm{II}}+\mu_{p o l}^{\mathrm{III}}+\mu_{p o l}^{\mathrm{IV}}+2 \mu_{p o l}^{\mathrm{V}},
$$

where

$$
\begin{gathered}
\mu_{p o l}^{\mathrm{I}}=T_{0} \varepsilon_{p o l}^{\mathrm{I}}, \quad \mu_{p o l}^{\mathrm{II}}=T_{1} \varepsilon_{p o l}^{\mathrm{II}}, \quad \mu_{p o l}^{\mathrm{III}}=R_{0 c} \varepsilon_{p o l}^{\mathrm{III}}, \\
\mu_{p o l}^{\mathrm{IV}}=R_{0 s} \varepsilon_{p o l}^{\mathrm{IV}}, \quad \mu_{p o l}^{\mathrm{V}}=R_{1} \varepsilon_{p o l}^{\mathrm{V}} .
\end{gathered}
$$

It follows immediately that, unlike for the Kelvin decomposition, eq. (11), $a$ polar stress can never be proportional to its corresponding polar strain; in fact, if one considers for instance the case of $\sigma_{p o l}^{\mathrm{I}}$ and $\varepsilon_{p o l}^{\mathrm{I}}$, it is

$$
\sigma_{p o l}^{\mathrm{I}}=\mu_{p o l}^{\mathrm{I}}=T_{0} \varepsilon_{p o l}^{\mathrm{I}} \Longleftrightarrow \sigma=T_{0} \varepsilon,
$$

which can never be true, also in the isotropic case, by virtue of (45), because the polar isotropic moduli $T_{0}$ and $T_{1}$ are strictly positive, [10]. This explains also, mechanically, why the polar stresses cannot decompose $\sigma$, eq. (59). A similar result is valid, of course, also for the polar strains.

In several cases considered in the past, the polar formalism has proved to be an effective method for handling problems concerning bi-dimensional anisotropy; some new phenomena and results have been obtained thanks to its use (for instance, the existence of the special $R_{0}$-orthotropy [11], the special orthotropy of paper [13], the pioneer theoretical work on anisotropy of complex bodies [14], the unexplored phenomena of interaction between geometry and anisotropy [17]).

As it has been recognized by de Saxcé and Vallée in [4], this has been the true leading method in the last decade for the analysis of plane anisotropy, and the results found thanks to the polar formalism have, at least in part, motivated a new interest in the study of bi-dimensional anisotropic elasticity, as the work of de Saxcé and Vallée shows. Nevertheless, the polar formalism has been applied to the study not only of elasticity, but also of piezoelectricity [18], of strength [19] and of complex bodies [14]. This has not yet been done with other theoretical approaches, while the results found 
with the polar formalism have, in some cases, motivated similar studies also in threedimensions, as for instance in the case of $R_{0}$-orthotropy in $\mathbb{R}^{3}$, whose existence has been confirmed by S. Forte [16], as said above, or the invariants of a third-order piezoelectric tensor, generalized to the 3D case by F. Ahmad, [20].

Of course, other approaches and formalisms exist for describing an anisotropic tensor and the polar method is not the oldest one. Some other approaches can be cited, besides the most recent one, the already cited work of de Saxcé and Vallée: the very classical formalism of Stroh [21], the works of Forte and Vianello [22], of Rychlewsky [6] and, for those who are familiar with the mechanics of composite materials, the so-called parameters of Tsai and Pagano [23].

A comparison between these last and the polar formalism has been already proposed in [10], while the comparison with the Stroh formalism, the works of Forte and Vianello or the representation of Rychlewsky is still to be done. Nevertheless, some points are intrinsic to the polar formalism and constitute, to the opinion of the authors, the key of the success of this approach in dealing with some problems, like those mentioned above or more generally in several works concerning the optimization of laminated anisotropic structures appeared in the last few years, see for instance [24], [25], [26]. To this purpose, it seems to the authors that the polar formalism has found much more and different applications to engineering problems than, for instance, the parameters of Tsai and Pagano, whose use is rather bounded to a certain class of problems, exclusively concerning the elasticity of laminates.

Namely, at its true origin the polar method is a mathematical technique to find the invariants of a tensor of any order in $\mathbb{R}^{2}$, so it is not at all bounded to the study of the elasticity tensor. A second fundamental point, distinguishing the polar formalism from other approaches, is that it does not makes use of only polynomial invariants: unlike other methods, the polar formalism looks for invariants linked to the direction. Its true peculiarity is to be based upon a representation of a tensorial quantity by modules and angles, for any tensorial order.

In this way, the direction enters directly and explicitly in the expression of the physical quantities, which is not the case with any of the other approaches mentioned above. The utility of such a result whenever the phenomenon to be described is directiondependent, which is of course always the case in anisotropy problems, is evident by itself.

Perspective: application of the polar decomposition to damage mechanics. The Kelvin decomposition has recently been used to define damage models using the Kelvin stresses as multiple effective stresses [8] or using the Kelvin decomposition of a fourthorder damage tensor [15]. One difficulty in such approaches is that for orthotropy, the Kelvin projectors cannot be defined independently of the material moduli. In this case, the polar decomposition could advantageously be used in $2 \mathrm{D}$ models for $R_{0}$-orthotropy, $r_{0}$-orthotropy and ordinary orthotropy (or even anisotropy) in order to define new damage models. That was the objective that mainly motivated the present research; this paper contains its first paths, hopefully preparing some new results to come. 


\section{References}

[1] W. Thomson (Lord Kelvin). Elements of a mathematical theory of elasticity. Philosophical Transactions of the Royal Society, 146:481-498, 1856.

[2] W. Voigt. Lehrbuch der Krystallphysik. Teubner, 1910.

[3] M.M. Mehrabadi and S.C. Cowin. Eigentensors of linear anisotropic elastic materials. The Quarterly Journal of Mechanics and Applied Mathematics, 43:15-41, 1990.

[4] G. de Saxcé and C. Vallée. Invariant measures of the lack of symmetry with respect to the symmetry groups of $2 \mathrm{~d}$ elasticity tensors. Journal of Elasticity, 111:21-39, 2013.

[5] M. E. Gurtin. An introduction to continuum mechanics. Academic Press, 1981.

[6] J. Rychlewsky. On Hooke's law. Prikladnaya Matematika i Mekhanika, 48:420435, 1984.

[7] M. François. Idéntification des symétries matérielles de matériaux anisotropes. $\mathrm{PhD}$ thesis, University Pierre et Marie Curie, Paris, 1995.

[8] R. Desmorat and R. Marull. Non-quadratic Kelvin modes based plasticity criteria for anisotropic materials. International Journal of Plasticity, 27:328-351, 2011.

[9] G. Verchery. Les invariants des tenseurs d'ordre 4 du type de l'élasticité. In Proc. of colloque Euromech 115, Villard-de-Lans, (France), 1979.

[10] P. Vannucci. Plane anisotropy by the polar method. Meccanica, 40:437-454, 2005.

[11] P. Vannucci. A special planar orthotropic material. Journal of Elasticity, 67:8196, 2002.

[12] P. Vannucci. Influence of invariant material parameters on the flexural optimal design of thin anisotropic laminates. International Journal of Mechanical Sciences, 51:192-203, 2009 .

[13] P. Vannucci. On special orthotropy of paper. Journal of Elasticity, 99:75-83, 2010.

[14] P. Vannucci and G. Verchery. Anisotropy of plane complex elastic bodies. International Journal of Solids and Structures, 47:1154-1166, 2010.

[15] M. François. A damage model based on Kelvin eigentensors and Curie principle. Mechanics of Materials, 44:23 - 34, 2012.

[16] S. Forte. Classi di simmetria in elasticità piana. In Proc. of 17th AIMETA Congress of Theoretical and Applied Mechanics - Florence, Italy, 2005. 
[17] P. Vannucci. Strange laminates. Mathematical Methods in the Applied Sciences, 35:1532-1546, 2012.

[18] P. Vannucci. The polar analysis of a third order piezoelectricity-like plane tensor. International Journal of Solids and Structures, 44:7803-7815, 2007.

[19] A. Catapano, B. Desmorat, and P. Vannucci. Invariant formulation of phenomenological failure criteria for orthotropic sheets and optimisation of their strength. Mathematical Methods in the Applied Sciences, 35:1842-1858, 2012.

[20] F. Ahmad. Invariants of a cartesian tensor of rank 3. Archive of Mechanics, 4:383-392, 2011.

[21] T. T. C. Ting. Anisotropic elasticity. 1996.

[22] S. Forte and M. Vianello. Symmetry classes for elasticity tensors. Journal of Elasticity, 43:81-108, 1996.

[23] S. W. Tsai and N. J. Pagano. Invariant properties of composite materials. In S. W. Tsai, J. C. Halpin, and N. J. Pagano, editors, Composite Materials Workshop, pages 233-253. Technomic, 1968.

[24] A. Jibawy, C. Julien, B. Desmorat, A. Vincenti, and F. Léné. Hierarchical structural optimization of laminated plates using polar representation. International Journal of Solids and Structures, 48:2576-2584, 2012.

[25] M. Montemurro, A. Vincenti, and P. Vannucci. A two-level procedure for the global optimum design of composite modular structures - Application to the design of an aircraft wing. Part 1: theoretical formulation. Journal of Optimization Theory and Applications, 155:1-23, 2012.

[26] M. Montemurro, A. Vincenti, and P. Vannucci. A two-level procedure for the global optimum design of composite modular structures - Application to the design of an aircraft wing. Part 2: numerical aspects and examples. Journal of Optimization Theory and Applications, 155:24-53, 2012. 